\title{
LA TRADUCCIÓN INGLESA DE HIPÓLITO Y AMINTA: UNA ADAPTACIÓN CON FINES COMERCIALES
}

\author{
María Rocío Lepe García \\ IES San Sebastián (Huelva) \\ roclroc@hotmail.com
}

L

as dos novelas de Francisco de Quintana, Experiencias de amor y fortuna (1626) y la Historia de Hipólito y Aminta (1627), disfrutaron de un notable éxito tanto en España como en el extranjero tras su publicación. Aparte de un conjunto importante de reimpresiones (Lepe García 2013: 78 y 123), las dos ficciones también fueron traducidas a otros idiomas. Experiencias de amor y fortuna primero al francés, La Fenise: histoire espagnole (París, 1636); después al inglés, The History of Don Fenise (1651) ${ }^{1}$; y más tarde al italiano, Il Feniso, ovvero Avvenimenti d'amore e di fortuna (1654)2, una traslación de la que se hace eco el bibliógrafo sevillano Nicolás Antonio: «quod opus Italice versum extat per Bartholo della Bella, Venetiis, anno 1654, in 12» (1996: 463). De las tres traducciones fue la versión inglesa la que alcanzó una mayor repercusión en la literatura extranjera, ya que, tal como indica el crítico Gerard Langbaine, la historia de Quintana alberga el germen de algunas novelas inglesas posteriores como Dutch Lover: A Comedy (Behn: 1673) y The Banditti or a Lady's Distress (D’Urfey: 1686). Igualmente testimonia la amplia difusión de esta novela la aparición del título en los catálogos de diferentes bibliotecas extranjeras como las pertenecientes a don Simón de Santander y a monseñor le Maréchal Duc d’Estrées ${ }^{3}$.

La segunda novela de Quintana, la Historia de Hipólito y Aminta, después de tres reediciones en el siglo XVII, fue también traducida al inglés un siglo después

\footnotetext{
1 Para Franklin P. Rolfe esta segunda traducción al inglés se realizó a partir de la anterior versión francesa, en Randall (1963: 118).

2 Esta traducción fue realizada por Bartolomé de Bella.

3 El interés actual por la novela queda de manifiesto en artículos diversos, como los escritos por Sánchez Escribano (1987: 189-204) y Bresadola (2011: 92-112), así como en la edición crítica y estudio realizados por este último (2012).
} 
de su primera publicación con el título The Most Entertaining History of Hippolyto and Aminta: Containing Great Variety of surprising Events in their Lives, and Those of Other Remarkable Persons. La traslación, llevada a cabo por John Stevens, fue impresa en los talleres de Arthur Bettesworth y Jeremiah Batley, en 1718, y reeditada poco después, en 1729. En este artículo pretendemos analizar los mecanismos empleados por el capitán Stevens en la traducción, así como advertir la intención de su técnica. Para el estudio hemos utilizado una reproducción de la segunda edición de la novela en $12^{\circ}$ con 395 páginas (4 preliminares más 391$)^{4}$.

Por razones de espacio, reducimos el análisis de las modificaciones textuales en sus diversos planos - superestructural, macroestructural y microestructural- a una observación de las alteraciones más significativas del primer nivel, integrando en el estudio las variaciones de los otros dos parámetros. Del acercamiento obtendremos algunas conclusiones sobre los procedimientos seguidos por el traductor —adición, supresión, permutación e inmutación一, así como su finalidad última.

Para empezar, la traducción inglesa de la Historia de Hipólito y Aminta modifica el armazón estructural del texto castellano. Comienza con una portada donde aparecen los datos principales de la obra y un prólogo del traductor ${ }^{5}$, elimina todos los textos preliminares de la fuente original y no segmenta la narración en ocho discursos, sino en nueve partes o capítulos traducidos con el vocablo inglés «book».

En la portada se aprecian las primeras novedades de la traslación. Esta consta de título «The Most Entertaining History of Hippolyto and Aminta»; una referencia al contenido de la novela «Great Variety of Surprising Events in their Lives, and Those of Other Remarkable Persons»; el índice de los capítulos en dos columnas y una breve referencia al contenido; las menciones al autor «Don Francisco de Quintana» y al editor «by Capt. Stevens»; el número de edición «the second edition»; y, por último, el lugar de la publicación «London», el nombre de los impresores «W. Bettesworth y J. Batley» y la fecha «1729». El aspecto más reseñable de la portada, aparte del catálogo de capítulos ${ }^{6}$, es el conjunto de valoraciones mayestáticas. El traductor añade al rótulo original, un adjetivo en grado superlativo «The most entertaining»; varios al subtítulo «great», «surprising», «remarkable»; y etiquetas efectistas al índice como «deep amorous intrigues», «strange exploits of valour», «miserable captivities» 0 «delightful tales». Estas manipulaciones, intencionadas sin duda, responden a la premisa clásica de la admiratio con un objetivo editorial muy definido. Su propósito principal, siguiendo los mecanismos

La reproducción corre a cargo de la editorial londinense British Library (Londres).

Aunque no está firmado, lo más probable es que la redacción del prólogo corresponda al mismo traductor de la novela.

6 Ninguna edición española hasta la fecha cuenta con un índice de contenidos. 
de mercadotecnia de una empresa, consiste en captar a un importante número de lectores y, por consiguiente, de compradores.

El prólogo o «preface» es un texto breve de dos páginas, de nueva redacción y de especial interés por las valoraciones y comentarios encomiásticos. El prologuista empieza destacando, como mérito principal de la ficción, el cumplimiento del precepto clásico «enseñar deleitando»: «This history is not only diverting, but instructive; it has all the air and gaity that can please youth and the fair sex, and yet it affords excellent morals for the more thinking and grave part of mankind» (Quintana 1729: prólogo), donde importa subrayar especialmente la referencia a los destinatarios de la novela, especialmente los jóvenes y las mujeres 7 . Continúa con una ponderación de los principales valores de la historia, entre los que destaca la eliminación de contenidos indecentes, el premio a la virtud, el carácter honorable del amor, la importancia del valor - siempre al servicio de la justicia y la inocencia-, el castigo a las acciones mezquinas y, sobre todo, que «everythig tends to the improvement of the reader» (1729: prólogo). El prefacio concluye con una mención al reconocimiento de Quintana en España y el deseo de que la novela obtenga la misma exitosa recepción en Inglaterra, ya que la traducción ha intentado preservar la belleza del original.

Dejando a un lado estas variaciones preliminares propiciadas por la campaña de marketing y la distancia temporal, el bloque más interesante de la traslación es la adaptación de la historia propiamente dicha. John Stevens se ajusta bastante a los ejes narrativos de la ficción, pero realiza modificaciones importantes en el texto que no se pueden soslayar. En primer lugar, y se trata probablemente de la alteración más destacada, añade un libro a la estructura axial de la edición española. Los ocho primeros capítulos de la versión inglesa coinciden básicamente con la historia base y el noveno es un apéndice sumado a la fábula en sustitución del poema la Descripción de la fiesta de Santiago el Verde.

En el plano tipográfico también se observa alguna modificación en las páginas de la traducción. El impresor, por ejemplo, encabeza y remata cada "book" con un elemento ornamental, igual que la cuarta edición española (1729) ${ }^{8}$, y marca el final de cada capítulo a partir del segundo libro con una fórmula fija: «The end of the second book ${ }^{9}$. Otras variaciones adicionales son el empleo de cursivas en los nombres propios; comillas inglesas o cursivas indistintamente en los diálogos

A ellos aluden Quintana en el prólogo de la novela (1627: f. 6v) y Gracián. Véase Baquero Goyanes (1983: 13-29) y González de Amezúa (1951: 255).

8 Los motivos, geométricos y florales en su mayor parte, coinciden en algunos casos con los incluidos en la edición de Pedro Joseph Alonso Padilla. El adorno figurativo del primer libro representa cuatro Cupidos en un jardín cortesano; el de los restantes libros es un mismo dibujo de trazado geométrico. Los remates, también muy parecidos entre sí, contienen aves y motivos florales en los libros primero, sexto y octavo y detalles geométricos en los demás.

$9 \quad$ En la fórmula solo varía el numeral ordinal.

Edad de Oro, XXXIII (2014), pp. 281-294, ISSN: 0212-0429 
en estilo directo; y un uso muy arbitrario de la letra mayúscula en los sustantivos, probablemente para fijar la atención del lector en los conceptos o ideas más relevantes.

Respecto a la traducción de la historia, el transcriptor respeta el hilo vertebral de la materia novelesca en sus pilares básicos, especialmente la estructura y las unidades narrativas, pero se percibe desde el principio en el trabajo un afán de simplificación, propósito que aumenta a medida que avanza la traducción. Basta para ello observar la progresiva reducción de páginas en cada capítulo a partir del tercer libro. ¿A qué se deben estos reajustes textuales? En nuestra opinión a motivos editoriales, como la necesidad de adaptar la producción a un proyecto y presupuesto iniciales establecidos en la fase previa a su cumplimiento. Contribuye a este planteamiento la inclusión de una segunda novela en el tomo, una novedad que responde a la técnica de marketing «dos por uno». Todos estos factores extraliterarios sostienen el proceso de traducción de John Stevens. Forzado por estos requisitos, como por el número de páginas preestablecido, el traductor vierte, adapta y reajusta el texto original a un nuevo molde, eliminando de la versión en inglés todos los detalles irrelevantes e innecesarios para el seguimiento de la historia. ¿Qué criterios sigue el traductor en esta discriminación? Sobre todo conservar el armazón argumental, mantener el interés del lector y favorecer la comprensión de la fábula. De acuerdo con estas pretensiones, el mecanismo que prevalece en el nuevo formato es la supresión, total en unos casos y parcial en otros.

La primera, la eliminación completa, es sistemática en las composiciones poéticas. El traductor suprime todos los poemas del texto originario sin excepción. Estas elisiones implican la realización de una serie de adaptaciones textuales en la versión traducida. El procedimiento, muy similar en todas las secuencias, consiste básicamente en la concreción del episodio, la depuración de detalles accesorios y alguna referencia circunstancial, si procede, al poema elidido. Veamos los casos: en la recreación del Beatus ille horaciano por boca de Leonardo durante la celebración de sus nupcias («Dichoso aquel que pasa»), el traductor describe el ambiente festivo de la escena, pero no se demora en los pormenores del evento ni alude siquiera al asunto de la canción; solo indica que Leonardo tocó y cantó, lo hizo con mucha habilidad y lució una voz melodiosa: «He play’d and sang to it, with so much skill and such a voices» (4). El traductor no solo suprime el poema en la secuencia, sino también los detalles ambientales y alguna digresión innecesaria. Poco después, durante el encuentro de Hipólito con Leonardo en el camino hacia Salamanca, vuelve a simplificar los detalles, centrándose únicamente en la acción y obviando los poemas amorosos: «He overtook a comly youth. [...] They saluted one another» (30). Su finalidad meramente referencial propicia la depuración del texto y un estilo sintáctico sencillo con predominio de oraciones simples, así como la eliminación de adjetivos y adverbios superfluos. Esta simplificación 
afecta igualmente a las oraciones compuestas, como se puede ver en el ejemplo: «When they understood, they were both for Salamanca, they held on their way together» (Quintana 1729: 30). Más adelante, en el discurso segundo, el traductor suprime los poemas amorosos de don Alejandro y don Carlos a sus amadas, aunque los menciona e incluso apunta el motivo compositivo: «we had carelessly left two copies of verses on the table», «the first she read was in praise of her sister, write by me, which caus'd some envy in her, till reading the other, which was of a different hand, she perceiv'd it spoke her own praises» (108). Al comienzo del discurso tercero elimina la escena preámbulo de la acción donde se incluyen los dos poemas inaugurales, lo mismo que ocurre al final del libro cuarto, donde la canción de don Carlos es reemplazada por un hiperónimo: «His relation being ended, they pass'd the time with other diversions» (206). En el libro quinto suprime nuevamente los dos poemas cantados en casa de Constanza, dejando en su lugar una simple alusión al festejo: «Some time was spent in hearing the pleasant music of voices and instruments» (212). Y en el discurso séptimo menciona la diversión, pero no incluye los poemas de don Carlos interpretados por Eusebio en el sarao: «We spent the day there with much satisfaction» (Quintana 1729: 270). En consecuencia, el procedimiento discursivo empleado en todas las secuencias es el resumen sintetizador con una querencia de la traducción hacia lo narrativo, a expensas del componente lírico.

Este mismo mecanismo simplificador puede observarse en las digresiones textuales, tanto iniciales como internas ${ }^{10}$. En el texto castellano las disertaciones iniciales están estrechamente interrelacionadas con la historia narrada a continuación. Sus funciones en el discurso son básicamente tres: organizar la estructura del relato, instruir en distintas materias y fijar la enseñanza moral ${ }^{11}$. El traductor otorga un tratamiento desigual a estos discursos inaugurales. Los transcribe de manera literal, prácticamente palabra por palabra, en los tres primeros libros; los omite completamente en los libros cuarto, sexto, séptimo y octavo; y, por último, reduce la reflexión inicial del libro quinto sobre la fortaleza de ánimo a una tercera parte. Una observación detenida del sistema empleado induce a plantearnos que la discriminación tampoco es casual, pues mantiene los discursos relacionados con los valores morales ${ }^{12}$ y elimina las pinceladas que tratan sobre cuestiones

10 Francisco Rico denomina «ingresiones» a las disertaciones iniciales de discurso (1967: 171-184). Los temas de estas reflexiones son la soledad (discurso primero), el valor (discurso segundo), la esperanza (discurso tercero), la prisión (discurso cuarto), la fortaleza de ánimo (discurso quinto), la nobleza social (discurso sexto), la pobreza (discurso séptimo) y la avaricia (discurso octavo).

11 No puede obviarse el prurito de notoriedad latente en estos discursos eruditos, aunque Quintana se muestra mucho más moderado que su maestro. Dejando a un lado esta cuestión, lo más probable es que el joven novelista empleara las mismas fuentes que Lope. Véase Bonilla Cerezo (2010).

12 Aunque también suprime las disertaciones iniciales sobre la pobreza y avaricia, susceptibles de conservación por su contenido. 
sociales. ¿Qué criterios inducen al traductor a conservar unas lucubraciones y a pulir o eliminar otras? No es necesario indagar demasiado para vislumbrar la intención inicial de conservar el texto original en su máxima pureza, eliminando el menor número de componentes, como puede comprobarse en los tres primeros libros. Sin embargo, la necesidad de ajustar el texto cada vez más a los requisitos tipográficos de la edición exige a John Stevens la eliminación de contenidos insignificantes, de aquí la omisión de la mayoría de las disquisiciones posteriores. También es posible conjeturar que por su ubicación en un contexto cultural -espacial y temporal- diferente, el autor no compartiera algunos de los argumentos esgrimidos por Quintana, aunque las razones que priman parecen ser editoriales.

Las digresiones internas del texto original se distinguen asimismo por la brevedad, aunque con distintos grados de concisión. Los excursos oscilan desde escuetos incisos que apostillan la narración hasta amplios intercolumnios ajenos a la historia. En el texto inglés vuelve a apreciarse en estas unidades la tendencia anterior a la reducción, bien mediante la eliminación completa de las intermisiones, bien por simplificación de las mismas. Aparte de los motivos de imprenta ya referidos, el transcriptor atiende también en estos casos a cuestiones narrativas. Como sabemos, la finalidad original de estos comentarios internos es preponderantemente moral. Quintana pretendía instruir al lector en los principios cristianos y el comportamiento humano. El traductor inglés, más interesado en publicar una historia que en adoctrinar a sus contemporáneos, procura pulir la fuente primigenia eliminando todo comentario trivial, descontextualizado o lejano de la moral inglesa contemporánea. Por estas razones, en la traducción se suprimen digresiones amplias como la crítica a los madrileños (discurso tercero), la disertación de Aminta sobre las propiedades de la luz (discurso quinto) o la reflexión de Hipólito sobre la pena capital (discurso octavo).

Como resulta impracticable un rastreo completo y exhaustivo de todas las digresiones internas, elegimos solo algunas muestras de cada uno de los procedimientos argumentativos empleados en estas reflexiones para extraer conclusiones posteriores sobre los mecanismos aplicados - la aceptación del inciso (con o sin cambio) o la supresión - y las causas de una u otra medida, tales como razones tipográficas, narrativas o estilísticas.

En principio, la tipología argumentativa empleada en las digresiones es muy variada. Pueden encontrarse argumentos de auctoritas, ad iudicium, analogía, anécdotas y ejemplos, interrogaciones y exclamaciones retóricas, alusiones a la propia experiencia personal, ad populum e incluso de refutación. Entre los primeros aflora la erudita digresión en el haber de Aminta sobre las mujeres para demostrar que «ni somos de diferente naturaleza que los hombres ni son menos perfectas, en cuanto a la perfección sustancial, nuestras almas» (Quintana 1627: f. 9v). En la relación de personajes insignes de la Antigüedad menciona a 
Hortensia, Corina, Aspasia, Zenobia, Cornelia, Eudoxia, entre otras mujeres destacadas. Hipólito aprovecha la digresión para incluir en la lista a Beatriz Galindo, la Latina ${ }^{13}$. El traductor elimina el párrafo completo en este pasaje para centrarse en los acontecimientos vitales de la protagonista. Más adelante, omite también la cita de Cicerón sobre los beneficios del estudio: «Los estudios alimentan en la mocedad, deleitan en la vejez, adornan en la prosperidad, ayudan en la adversidad, anochecen con nosotros, peregrinan en nuestra compañía y, aun entre la rusticidad del campo, no nos desamparan» (Quintana 1627: f. 34r); sin embargo, poco después mantiene la extensa disertación de don Carlos sobre la amistad a partir de «these precepts, and tho' they come from my mouth, relieve you hear them from Seneca, Cicero, and Quintilian, whose they are originally» (Quintana 1729: 90). En estos contextos, el narrador elimina las dos primeras citas y mantiene la tercera por razones meramente narrativas, ya que la última es fundamental para entender los acontecimientos posteriores y las anteriores no son coadyuvantes ${ }^{14}$. El criterio general, pues, en la traducción se antoja la supresión de las citas eruditas ${ }^{15}$.

La misma tendencia a la elisión se aprecia en los argumentos ad iudicium, muy abundantes en el texto original. Quintana suele emplearlos en las digresiones breves, especialmente en las coletillas de los episodios o asuntos. El traductor parece preferir la omisión de estos argumentos por no representar su propio pensamiento y entorpecer el ritmo de la narración. Entre las numerosísimas apostillas suprimidas en la versión inglesa extraemos como ejemplo la siguiente: «Nunca dejan de manifestarse en las palabras o en las acciones los más ocultos deseos si no se miden con singular cuidado» (Quintana 1627: f. 113r), para referir las intenciones de don Alonso y don Juan de salir a las fiestas de Alcalá de manera furtiva, dejando a Hipólito en casa.

En las digresiones por analogía o asociación no existe, en cambio, una pauta fija. El traductor opta por la conservación en unos casos, como la reflexión sobre los favores: «in the mouth of a bee produces honey and in that of spider venome» (Quintana 1729: 23); y en cambio cercena otras como el ejemplo histórico de la batalla de Demetrio contra Ptolomeo ${ }^{16}$ (Quintana 1627: f. 78r) para insistir en la necesidad de prevención y prudencia en todas las acciones humanas.

En la novela fuente, aparte de este episodio, se incluyen un par de anécdotas históricas para amenizar el relato y apoyar el mensaje del texto: la costumbre punitiva de los etruscos (discurso primero) y uno de los dichos del rey don Alonso de

13 Sobre Beatriz Galindo, véase María Teresa Álvarez (2003: 69 y ss.)

14 Un lector inglés del siglo XVIII desconocía seguramente quién era Beatriz Galindo y, por otro lado, la cita de Cicerón carece de interés narrativo.

15 El traductor elimina también las que aparecen al principio de los libros quinto, séptimo y octavo.

16 La anécdota histórica, el enfrentamiento en Gaza entre Demetrio I, rey de Macedonia, y Ptolomeo I, fundador de la dinastía Ptolemaica en el año 312 a. C., advierte sobre la necesidad de ser prudente y prevenir riesgos irreparables. 
Aragón (discurso quinto). El primero se inserta en la diatriba de Lupercio contra los matrimonios impuestos. Para sustentar su argumentación, el personaje refiere la macabra tradición de este pueblo toscano: «Antiguamente solían los etruscos, gente bárbara para castigar los delitos en que intervenían dos, matar a el uno y atar al ya frío cadáver el otro, para que el mal olor le acabase haciendo instrumento de su castigo al mismo que fue su compañero en los yerros» (Quintana 1627: f. 22r). En este caso el traductor recoge la referencia histórica, pero la simplifica (Quintana 1729: 42). En el segundo, sin embargo, opta por la supresión completa (discurso quinto $)^{17}$, entre otras razones porque elimina asimismo la ingresión donde se inserta. La arbitrariedad atiende en esta secuencia a factores tipográficos, narrativos y contextuales. Primero, la tendencia a la elipsis aumenta a medida que avanza la traducción, como ya se ha apuntado; segundo, la primera referencia histórica forma parte de un discurso fundamental en el avance de la trama; y, tercero, la segunda anécdota resulta de escaso interés para un lector inglés de aquel tiempo. El redactor intenta, además de traducir, modernizar la historia y acercarla a los lectores de su época.

La voz del narrador se percibe asimismo en los recursos patéticos, como son las numerosas interrogaciones y exclamaciones retóricas diseminadas por el texto original con el fin de despertar emociones diversas en el lector. Aparecen sobre todo en las reflexiones que versan sobre el comportamiento y los sentimientos humanos. Como los ejemplos son numerosos, hemos elegido para el caso un par de muestras; la elucubración sobre el ejercicio de la imaginación en el enamoramiento de Marcela:

¿Quién no advierte en este suceso el justo castigo que ordinariamente se sigue a la presunción y un ejemplar vivo de los medios con que tal vez se postra la vanidad? ¿Quién no vio a Marcela libre a muchos amores verdaderos y la ve sujeta a una imaginación? ¿Quién no la vio blasonar de rendir a todos y la ve no avergonzarse de estar rendida a una imagen de su idea? (Quintana 1627: f. 51r).

Y el discurso patético de don Carlos sobre la amistad. En el primer caso, el traductor suprime el conjunto de interrogaciones retóricas, sintetizando la idea primigenia en una exposición más concisa: «She began to consider, who the author might be, and to describe in her fancy such a one as she could with. She form'd in her idea a brave man, of good sense, discreet, handsome, [...] having fram'd such an object, she began to love it» (Quintana 1729: 108). En el ejemplo anterior pueden apreciarse la sencillez y linealidad sintácticas de las oraciones. En la

17 La anécdota pertenece al rey don Alonso I de Aragón y V de Nápoles. El comentario, una defensa de la virtud y el valor personales sobre la herencia, se encuentra en el Libro de los dichos (1527: II, f. XXVIIv). 
disertación de don Carlos, en cambio, el autor conserva la cadena de interrogaciones retóricas aligerando únicamente el número. De las ocho enlazadas en el texto castellano, el traductor reproduce únicamente seis. ¿Qué criterio sigue el traductor en la adaptación? Evidentemente narrativo. John Stevens pretende ofrecer una historia fluida y entretenida a los lectores, obviando todo aquello que distraiga la atención de la peripecia narrativa.

Otra modalidad argumentativa recurrente en las digresiones es la apelación a la propia experiencia. Siguiendo la tendencia dominante, el traductor también se inclina por la eliminación o simplificación del recurso. Así, elimina la censura de Jacinto sobre los castigos inmoderados de los padres a sus hijos: «porque veáis la moderación con que se deben castigar los hijos y lo poco que se remedia cuando el rigor es desigual a la culpa» (Quintana 1729: 175). Y conserva, muy simplificada, la disertación final de Aminta sobre su intención de ingresar en un convento. El transcriptor no anula el discurso en el segundo caso por su importancia narrativa, pero filtra el mensaje hasta dejarlo reducido a su esencia.

Algo parecido ocurre en las digresiones ad populum de contenido religioso o patriótico, como la apología del clero y la diatriba contra los arbitristas. Respecto a la primera, el traductor plasma en la versión inglesa solo el contenido de la reflexión: «Hippolyto boldly floyd up in defence of the christian goverment» (Quintana 1729: 237); y respecto a la segunda, la omite completamente (Quintana 1627: ff. 58v-61v).

Finalmente, las dos digresiones de refutación u oposición contenidas en el texto castellano - la diatriba familiar sobre el matrimonio de Feliciana (Quintana 1627: 21v) y la dialéctica contra la astrología judiciaria (Quintana 1627: ff. 137v138) - aparecen prácticamente completas en la versión inglesa. La conservación de las dos disertaciones responde nuevamente a motivos narrativos. Ambas son necesarias para la progresión de la acción: la primera conduce al matrimonio desgraciado de Feliciana y la segunda al encarcelamiento de Hipólito.

Por último, el traductor también evita innumerables ejemplos de omnisciencia discursiva bien de duda o valoración. Elegimos entre otros ejemplos los siguientes: «Amaba el anciano don Pedro tiernamente a doña Clara, su hija menor, o ya porque el serlo le obligaba o ya porque su cordura, su apacibilidad y su hermosura granjeaban justísimamente tanto amor» (Quintana 1627: f. 90v); «Alí, entonces, o por condescender a los ruegos de su hermana o por tener con su ayuda más comodidad para avisarle de lo que pasaba, la manifestó las desdichas de Hipólito y las obligaciones que le reconocía por maestro y por amigo» (f. 141r). En ambos casos el traductor omite los comentarios por parecerles innecesarios desde el punto de vista narrativo, despejando el discurso de cualquier divagación. 
Por otra parte, aunque el capitán Stevens sigue escrupulosamente los episodios del texto castellano con bastante exactitud, en un caso aislado recrea la versión primitiva a favor de la concisión. La inmutatio se produce durante el episodio del cautiverio en Constantinopla. Alí ayuda a los protagonistas a salir de la celda donde se encuentran prisioneros, pero durante la huida se observa un cambio. En la versión inglesa, Aminta sale de la mazmorra por su propio pie, mientras que en el texto castellano es Hipólito quien, tras el desmayo de la joven, la ayuda a salir en brazos: «and Aminta having the key to it, she was first turn'd out that way» (Quintana 1729: 254).

Dejando a un lado todas estas manipulaciones textuales, el aspecto más novedoso de la traducción es la adenda de un nuevo libro (Book IX). El traductor suprime el poema colofón de la Descripción de las fiestas de Santiago el Verde, incluye en su lugar una novela corta y modifica la secuencia de engarce para hilar los dos relatos. Las variaciones que se observan en la secuencia puente del texto original son escasas. El narrador simplifica el discurso de Aminta y elimina completamente el encomio de Alejandro sobre la sabiduría de su hermana, aun conservando otras referencias, como el bautismo de Lidora y el itinerario posterior de los personajes: las bodas múltiples de las parejas de enamorados; el regreso de Leonardo y Feliciana a Barcelona; la llegada de Rezuán a Madrid un año más tarde, su conversión y bautismo; la marcha de don Alonso a Malta con don Juan para servir en la orden religiosa; y, por último, la permanencia de Hipólito en Madrid junto a su familia.

A continuación, el narrador añade una secuencia de enlace para introducir la tabla de novelas de Salas Barbadillo Corrección de vicios (1615). El entorno festivo de la celebración de las bodas se constituye en pórtico de la nueva novela. El pretexto recuerda sobremanera a las cornices de las colecciones de novela corta donde la reunión festiva permitía la inserción yuxtapuesta de un conjunto de relatos breves ${ }^{18}$. El traductor describe la celebración de las nupcias «The nuptials above mention'd were celebrated with all the magnificence that so solemn occasion an requir'd» (Quintana 1729: 306) y prolonga el festejo a lo largo de una semana con «innocents diversions» (307) como actuaciones teatrales, representadas por los comediantes del rey durante tres días, y conversaciones y músicas sobre asuntos divertidos, propios o de amigos, los restantes días. También Stevens introduce al autor de la novela como personaje de la ficción:

\footnotetext{
Alonso de Castillo Solórzano quizá sea el narrador contemporáneo con un empleo mayor de motivos festivos como marco en las colecciones de novelas: celebración de los Carnavales en Tiempo de regocijo (1627) y Sala de recreación (1649), la Nochebuena en Noches de placer (1621) y la Epifanía en Huerta de Valencia (1629). A estos asuntos hay que añadir otros como la curación de una enfermedad en Tardes entretenidas (1625) y Alivios de Casandra (1640), el viaje en Jornadas alegres (1626) o el retiro en La quinta de Laura, 1649. Véase Lepe García (2007).
} 
«Among the rest of the gentleman [...] was don Alonso de Salas, a native of Madrid, and a person of good natural and acquir'd parts, who being made sensible that something was expected from him for the public diversion, began as follows, in the next book» (308). El criterio de traducción seguido en esta segunda novela es el mismo que hemos apuntado para la Historia de Hipólito y Aminta: la conservación de los componentes narrativos básicos, la eliminación de todo asunto irrelevante e innecesario y la adaptación del verso a la prosa, como sucede en el relato de El mal fin de Juan de Buenalma. La novela de Salas Barbadillo, igual que sucede en las colecciones de novela corta, acaba con el aplauso y el regocijo de todos los presentes. De manera que la traducción inglesa de John Stevens entronca, por todos estos rasgos - la incorporación de un marco narrativo, la yuxtaposición de los relatos, la presencia de narratarios y la supuesta oralidad ${ }^{19}$ - , con el modelo genérico de la novela cortesana. De hecho, la misma creación de Quintana guarda igualmente ciertas similitudes con este venero áureo ${ }^{20}$. En consecuencia, la elección del editor resulta en este caso más que acertada. En nuestra opinión, la medida editorial de publicar dos novelas en un único volumen constituye asimismo una medida ventajosa para todos, ya que beneficia a la vez al editor y a los lectores.

Por último, respecto a los mecanismos lingüísticos empleados en la traducción, el capitán John Stevens transcribe en líneas generales con bastante exactitud el texto original. Siguiendo la gramática inglesa, las oraciones perstan un orden establecido con preferencia por la síntesis y eliminación. Esta depuración afecta unas veces a párrafos completos y otras a unidades oracionales. El purismo del transcriptor puede apreciarse en la siguiente traslación: «y este conociese a Laurencio, un criado que había tenido en Bolonia, y que como después sabremos, había ocasionado los disgustos de Aminta» (Quintana 1627: f. 65r); «the latter discover'd it was Laurencio, a servant he once had in Bolognia, and who as we shall be inform'd hereafter, had been the occasion of Aminta's misfortunes» (Quintana 1729: 135). La pulcritud, el celo y esmero son más que evidentes en esta referencia, pero en la mayoría de los casos sucede lo propio. En otros contextos oracionales parafrasea la fuente y emplea sinónimos con sentido similar. No es necesario espigar demasiado para probar lo comentado: «Don Henry hearing these words willingly have given him the finishing stroke to be the more sure of revenging his wrong» (Quintana 1729: 136); «Oyendo semejantes razones, don Enrique quiso acabar de matarle para asegurar más la venganza de su injuria» (Quintana 1627: f. 65v).

En definitiva, la traducción inglesa de la Historia de Hipólito y Aminta resulta un modelo de adaptación con fines comerciales. El traductor, siguiendo las

19 El narrador se dirige a los contertulios «began as follows in the next book» (Quintana 1729: 308).

20 Véase Lepe García (2013a: 291).

Edad de Oro, XXXIII (2014), pp. 281-294, ISSN: 0212-0429 
directrices de la empresa editorial, adecua el texto castellano a un nuevo molde fijado de antemano según unas pautas de edición. La sustitución del poema final Descripción de la fiesta de Santiago el Verde por un conjunto de novelas de Salas Barbadillo corrobora el interés comercial de la publicación, de igual modo que la reproducción en $12^{\circ}$, un tamaño propio de las ediciones de bolsillo más económicas y, por tanto, más vendibles. Con estos condicionantes comerciales, el traductor, aun conservando el texto primitivo en su máxima pureza, se ve obligado a depurar la fuente novelística para encajar las dos obras en un nuevo formato prefijado. Sus premisas, pues, son la conservación de las líneas axiales de la historia novelesca y la eliminación de todo lo accesorio como poemas, digresiones moralizantes, anécdotas, retoricismos, etc. Su labor, no obstante, resulta bastante cuidada y purista, ya que la discriminación se realiza con bastante acierto. Podría decirse que Stevens era un buen químico, capaz de destilar en el laboratorio de su estudio la sustancia volátil de lo huero y baladí de la materia contundente de la narración. Con toda seguridad, la primera traducción inglesa de la Historia de Hipólito y Aminta (1718) debió de gozar de un éxito estimable entre el público anglosajón de la época, ya que sin dicho favor no se hubiera reeditado solo once años más tarde.

Recibido: 17/09/2014

Aceptado: 30/10/2014

\section{OBRAS CITADAS}

Alonso Seodne, María José (1999). «Traducciones de obras narrativas en el Diario de Madrid, 1814-1820». En Francisco Lafarga Maduell (coord.), La traducción en España (1750-1830). Lengua, literatura, cultura. Lleida: Universitat de Lleida, pp. 363-374.

Álvarez García, María Teresa (2003). Ellas mismas. Madrid: La esfera de los libros.

AnTONIO, Nicolás (1996). Bibliotheca Hispana Nova. Madrid: Visor.

Baquero Goyanes, Mariano (1983). «Comedia y novela en el siglo XVII». En Serta philologica in Honorem Lázaro Carreter. Madrid: Cátedra, pp. 13-29.

Beccadelli, Antonio (1527). Libro de los dichos y hechos del rey don Alonso: ahora nuevamente traducido. http://parnaseo.uv.es/Lemir/textos/Dichos/Epistola.htm

Bonilla Cerezo, Rafael (2010). Novelas cortas del siglo XVII. Madrid: Cátedra.

Bresadola, Andrea (2011). «Elementos teatrales en Experiencias de amor y fortuna de Francisco de Quintana». En Luciana Gentilli y Renata Londero (eds.), Emocionar escribiendo. Teatralidad y géneros literarios en la España áurea. Madrid / Frankfurt: Iberoamericana - Vervuert, pp. 92-112.

Chenot, Beatriz (1982). «Vie madrilène et roman byzantin dans l'oeuvre de Francisco de Quintana». En Maxime Chevalier (ed.), Traditions populaires et diffusion de la culture en Espagne (XVI-XVII siècles). Burdeos: Presses Universitaires, pp. 131-148. 
GonZÁlez de AmezúA, Agustín (1951). «Formación y elementos de la novela cortesana». En Opúsculos histórico literarios. Madrid: CSIC, vol. I, pp. 194-279.

Lepe García, María Rocío (2007). Castillo Solórzano y la novela corta: Los alivios de Casandra y La quinta de Laura. Huelva: Universidad de Huelva.

(2013a). Historia de Hipólito y Aminta de Francisco de Quintana: estudio y edición crítica [tesis doctoral]. Huelva: Universidad de Huelva.

(2013b). «La Historia de Hipólito y Aminta de Francisco de Quintana: fuentes y modelos genéricos». Etiópicas, 9, pp. 261-352.

Quintana, Francisco de (1626). Experiencias de amor y fortuna. Madrid: Viuda de Alonso Martín.

(1627). Historia de Hipólito y Aminta. Madrid: viuda de Luis Sánchez.

(1729). The Most Entertaining History of Hippolyto and Aminta. Containing Great Variety of Surprising Events in Their Lives. Capt. Stevens (trad.). Londres: Arthur Bettesworth and Jeremiah Batley. (2012). Experiencias de amor y fortuna. Andrea Bresadola (ed.). Sevilla: UNIA.

Randall, Dale B. J. (1963). The Golden Tapestry. Durham: Duke University Press.

Rico, Francisco (1967). «Estructuras y reflejos de estructuras en el Guzmán de Alfarache». Modern Language Notes, 82, pp. 171-184.

Salas Barbadillo, Alonso Jerónimo de (1615). Corrección de vicios: en que Boca de todas verdades toma las armas contra la malicia de los vicios y descubre los caminos que guían la virtud. Madrid: Juan de la Cuesta.

SÁnchez Escribano, Francisco Javier (1987). «La versión inglesa de Experiencias de amor y fortuna, de Francisco de Quintana (1651)». En Julio César Santoyo Mediavilla e Isabel Verdaguer (eds.), De clásicos y traducciones: versiones inglesas de clásicos españoles (sS. XVI-XVII). Barcelona: PPU. 
LA TRADUCCIÓN INGLESA DE HIPÓLITO Y AMINTA: UNA ADAPTACIÓN CON FINES COMERCIALES

RESUMEN: Este artículo analiza los mecanismos de traducción en la versión inglesa de la Historia de Hipólito y Aminta de Francisco de Quintana (1627). La edición, realizada por John Stevens, fue publicada en 1718 y reeditada poco después, en 1729. El traductor respeta con bastante pulcritud en su trabajo las líneas narrativas básicas de la ficción, pero tiende continuamente a la simplificación, en aras de la brevedad. Este trabajo estudia los procedimientos empleados por el transcriptor en los diversos planos estructurales de la obra y valora la influencia de los factores comerciales en su conformación final.

Palabras clave: Francisco de Quintana, Historia de Hipólito y Aminta, Alonso de Salas Barbadillo, Corrección de vicios, Traducción inglesa, Mecanismos de traducción.

\section{The English Translation of Hippolytus and Aminta: an AdAPTATION FOR COMMERCIAL PURPOSES}

Aвstract: This article analyzes the translation mechanisms in the English version of the History of Hippolytus and Aminta written by Francisco Quintana (1627). The edition, carried out by John Stevens, was published in 1718 and reissued soon after in 1729. In this work, the translator respects the basic story lines of fiction fairly closely, but continuously tends to simplification in the interest of brevity. This paper studies the procedures used by the writer in various structural drawings of the work and also assess the influence of commercial factors in its final conformation.

Keywords: Francisco de Quintana, History of Hippolytus and Aminta, Alonso de Salas Barbadillo, Correction of bad habits, English Translation, Translation Mechanisms. 
Evangelina Rodríguez Cuadros (Universitat de València)

Novela cortesana, novela barroca, novela corta: de la incertidumbre al canon .9

Mita Valvassori (Universidad de Los Lagos)

El modelo narrativo del Decamerón en la Edad de Oro: una vieja historia .21

Antonio Gargano (Università degli Studi di Napoli Federico II)

«Difficile est proprie communia dicere»: el género de la novella entre

Boccaccio y Cervantes

Guillermo Carrascón (Università degli Studi di Torino)

Apuntes para un estudio de la presencia de Bandello en la

novela corta del siglo XVII

Leonardo Coppola (Università degli Studi «G. d'Annunzio» di Chieti-Pescara)

La proyección de Straparola en la novela española del Siglo de Oro desde una perspectiva editorial

Mireia Aldomì García

Didactismo, género literario y lector en Giraldi Cinzio.

María Jesús Zamora (Universidad Autónoma de Madrid)

«...En tiempo menos discreto que el de agora, aunque de hombres más sabios, se

Ilamaban a las novelas cuentos». La novela corta y el cuento en el Siglo de Oro.....109

Marcial Rubio (Università degli Studi «G. d'Annunzio» di Chieti-Pescara)

La contribución de Cervantes a la novela barroca: la ejemplaridad. .125

PIERRe Darnis (Université Bordeaux Montaigne)

La fuerza de la sangre, La ilustre fregona $y$ Las dos doncellas: ¿tres tipos

folclóricos?

María Soledad ArRedondo (Universidad Complutense de Madrid)

De La gitanilla $a$ La sabia Flora malsabidilla. El género, el personaje

y el matrimonio

Antonella Gallo (Università degli Studi di Verona)

Fabulaciones en equívocos burlescos: la Chrónica del monstro imaginado (1615)

de Alonso de Ledesma y novela corta barroca

David GonZález Ramírez (Universidad de Málaga)

El filósofo del aldea (1625) de Baltasar Mateo Velázquez: recepción textual

e hipótesis autorial.

Jonathan BRAdBury (University of Exeter)

La narrativa breve en la miscelánea del siglo XVII 
Cristina Castillo Martínez (Universidad de Jaén)

«La fuente del desengaño»: de las Noches de invierno de Eslava a la Tercera

Diana de Tejeda.

María Zerari (Université Paris-Sorbonne, CLEA)

Furor in fabula: La cruel aragonesa de Castillo Solórzano (o de la dama monstruo).. 241

Giulia Giorgi (Università degli Studi di Ferrara)

Alonso de Castillo Solórzano reescritor de sí mismo: algunas notas sobre los

Escarmientos de amor moralizados y el Lisardo enamorado .257

Angela Fabris (Alpen-Adria-Universität Klagenfurt)

El diálogo con el público y los espacios reales y de maravilla en

Casos prodigiosos y cueva encantada de Juan de Piña .267

María Rocío LePe García (IES San Sebastián, Huelva)

La traducción inglesa de Hipólito y Aminta: una adaptación

con fines comerciales 281

Andrea Bresadola (Università degli Studi di Udine)

La novela española en la Italia del siglo XVII: el caso de Il Feniso

de Francisco de Quintana

José Teruel (Universidad Autónoma de Madrid)

El triunfo del Desengaño. Marco y desengaño postrero de la Parte segunda

del Sarao y entretenimiento honesto, de María de Zayas

Nieves Romero-Díaz (Mount Holyoke College)

Lecturas alternativas en la Novela del fin bueno en mal principio

de doña Ana Francisca Abarca de Bolea.

Shifra Armon (University of Florida)

Compromiso y distanciamiento en La Venus de Ferrara

de Mariana de Carvajal Saavedra

Mechthild Albert (Rheinische Friedrich-Wilhelms-Universität Bonn)

Las "noches": un subgénero novelístico en perspectiva comparada.... .365

Fernando Copello Jouanchin (Université du Maine, Le Mans)

El mueble en la novela corta del Siglo de Oro: algunas reflexiones

en torno a la cama

Ilaria Resta (Università del Salento):

De la novella al entremés pasando por la novela corta: reescrituras del cuento

La gara delle tre mogli del Cieco di Ferrara. 


\section{EDAD DE ORO}

Revista de Filología Hispánica XXXIII

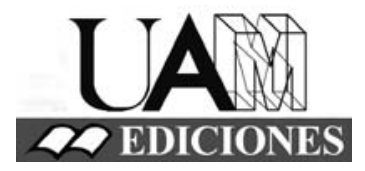




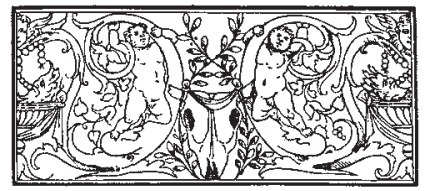

\section{Edad de Oro. Revista de Filología Hispánica}

ISSN: 0212-0429

Dirección:

Teodosio Fernández

Secretaría y edición:

José Ramón Trujillo

Coordinador del volumen XXXIII:

Rafael Bonilla Cerezo

Comité científico internacional:

Carlos Alvar (Univ. de Ginebra)

Ignacio Arellano (Univ. de Navarra)

Javier Blasco (Univ. de Valladolid)

Alberto Blecua (UAB)

Jean Canavaggio (Univ. de París X)

Laura Dolfi (Univ. de Turín)

Aurora Egido (Univ. de Zaragoza)

Víctor García de la Concha (RAE)

Luciano García Lorenzo (CSIC)

Joaquín González Cuenca (Univ. de Castilla-

La Mancha)

Agustín de La Granja (Univ. de Granada)

Begoña López Bueno (Univ. de Sevilla)

Michel Moner (Univ. de Toulouse III)

Joan Oleza (Univ. de Valencia)

Alfonso Rey (Univ. de Santiago)

Lina Rodríguez Cacho (Univ. de Salamanca)

Leonardo Romero Tobar (Univ. de Zaragoza)

Aldo Ruffinatto (Univ. de Turín)

Lía Schwartz (City University of New York)
Redacción y admisión de originales:

Teodosio Fernández

Edad de Oro

Departamento de Filología Española

Universidad Autónoma de Madrid

28049 Madrid (España)

Tfno.: +0034 914974090

correo: teodosio.fernandez@uam.es

Distribución, suscripción y venta:

Servicio de Publicaciones de la UAM

Universidad Autónoma de Madrid

28049 Madrid (España)

Intercambio de publicaciones:

Biblioteca de la Facultad de Filosofía y

Letras (UAM)

Universidad Autónoma de Madrid

28049 Madrid (España)

Han colaborado en este volumen:

Departamento de Filología Española (UAM)

Facultad de Filosofía y Letras (UAM)

Proyecto I+D FFI2013-41264-P La novela

corta del siglo XVII: estudio y edición (y II)

Edad de Oro se recoge en las siguientes bases de datos: SCOPUS, MLA Database, HLAS, Latindex, PIO-Periodical Content Index, ISOC, Dialnet, MIAR, ERIH, DICE, Sumaris CBUC, Ulrich's. Se encuentra evaluada en CIRC: A; INRECH: primer cuartil, posición 6 de 50; MIAR difusión ICDS live: 9.977; SCImago Journal \& Country Rank: H Index 2, SJR 0,101, Q4; RESH índice de impacto: 0.162; ERIH: A INT1; Carhus Plus+: B. 